\title{
CONVEX COMBINATIONS OF UNIFORMLY MEAN STABLE MARKOV OPERATORS
}

\author{
ROBERT SINE 1
}

ABSTRACT. A convex combination of commuting uniformly mean stable Markov operators acting on $C(X)$ is shown to be uniformly mean stable. The proof is completely geometric.

Let $C(X)$ denote the $B$-space of continuous functions on a compact Hausdorff space. A (stable) Markov operator $T$ is a positive ( $T f \geq 0$ whenever $f \geq 0$ ) linear transformation with $T 1=1$. It is immediate that if $S_{i}$ are Markov and $a_{i} \geq 0$ with $\sum a_{i}=1$ for $1 \leq i \leq n$ then $T=\sum a_{i} S_{i}$ is again a Markov operator. We call any Markov operator $T$ uniformly mean stable (u.m.s.) if the Cesàro means

$$
A_{n}(T) f=(1 / n)\left(I+T+\cdots+T^{n}\right) f
$$

converge uniformly for every $f$ in $C(X)$ (to a then necessarily continuous and invariant function). This strong convergence property is of interest in itself; moreover there is also a rather a complete ergodic decomposition for such operators (see [1], [2], [4], and [5]). Thus conditions which imply u.m.s. are of interest. For conditions which imply u.m.s. in the special cases of random point maps and random isometries, see [1] and the references given there. A more general classical result is that if $T$ is uniquely ergodic (that is $T$ has exactly one invariant probability) then $T$ is u.m.s. (This may be found in Oxtoby's well-known review [3] for point maps.) This result, in turn, suggested the following $[5$, Theorem 2.2 , but read $\neq$ for the misprint $=$ there] which we will need here: $T$ is u.m.s. if and only if the invariant functions of $T$ separate the invariant probabilities of $T$. (This has been generalized to contractions on arbitrary $B$-spaces as well [6].) We will prove the

Received by the editors March 18, 1974 and in revised form, May 14, 1974. AMS (MOS) subject classifications (1970). Primary 47 A35, 60J05.

Key words and phrases. Uniformly mean stable, Markov operators, convex combinations.

1 Partially supported by National Science. Foundation Grant \#GU 3171. 
Theorem. The convex combination of commuting u.m.s. Markov operators is u.m.s.

We make the observation here that it is sufficient to consider the convex combination of two operators. Thus we will take

$$
T=\alpha R+\beta S
$$

where $\alpha$ and $\beta$ are nonnegative and sum to 1 while $R$ and $S$ are commuting Markov operators. The set of invariant probabilities of $T$ will be denoted by $K(T)$ and similarly for $R$ and $S$. Observe that these sets are each nonempty by almost anyone's fixed point theorem and, moreover, $K(R) \cap K(S) \neq \varnothing$ by almost everyone's (Riesz-Kakutani-Markov) fixed point theorem.

Lemma. Let $T$ be a convex combination of two commuting u.m.s. Markov operators. Then $T$ is u.m.s. if and only if $K(T)=K(R) \cap K(S)$.

Proof. We clearly have $K(T) \supset K(R) \cap K(S)$ in every case. Suppose first we have $K(T)=K(R) \cap K(S)$. Let $\mu_{1}$ and $\mu_{2}$ be two distinct $T$-invariant probabilities. Then since $R$ is u.m.s and $\mu_{1}$ and $\mu_{2}$ are both in $K(R)$ there is a continuous $R$-invariant function $f$ with $\left(f, \mu_{1}\right) \leq c<d \leq\left(f, \mu_{2}\right)$. Since $S$ is u.m.s. we obtain a continuous limit by $g=\lim A_{n}(S) f$ where $g$ is both $R$ and $S$ invariant. But then $g$ is $T$-invariant and $\left(g, \mu_{1}\right) \leq c<d \leq$ $\left(g, \mu_{2}\right)$. Since the $T$-invariant functions separate the $T$-invariant probabilities, $T$ is u.m.s.

Now assume $T$ is u.m.s, and let $\mu$ be a $T$-invariant probability. If $\mu$ is not in $K(R) \cap K(S)$ we use the geometric Hahn-Banach theorem to obtain a continuous function $f$ so that for $\lambda$ in $K(R) \cap K(S)$ we have $(f, \lambda) \leq c<$ $d=(f, \mu)$. Let $g=\lim A_{n}(T) f$ and the same inequality will then hold for the $T$-invariant function $g$. Since $\int g d \mu=d$ the maximum of $g$ on $X$ is at least $d$. Let $M$ be the maximizing set of $g$. That is $M=\{x: g(x)=\sup g\}$.

We call any closed set $D$ with the property $T^{*} \delta(x)$ is supported in $D$ for all $x$ in $D$ an invariant set (= self-supporting in [5]). Then $M$ is a $T$ invariant set. But any $T$-invariant set is both $R$ and $S$ invariant as well. It follows at once that $M$ supports a probability, say $\sigma$, that is both $R$ and $S$ invariant. Now $(g, \sigma) \leq c<d \leq(g, \sigma)$ and this contradiction shows $K(T) \subset$ $K(R) \cap K(S)$ and thus finishes the proof.

Before using the Lemma to establish the Theorem we will make a comment using the Lemma in reverse. If $R$ and $S$ are commuting Markov matrices and $T=\alpha R+\beta S$ then $K(T)=K(R) \cap K(S)$. This is because of the well-known fact that all Markov matrices are u.m.s. 
Proof of the Theorem. Let $\lambda$ be an extreme point of the compact convex set $K(T)$. We observe that $T^{*} R^{*} \lambda=R^{*} T^{*} \lambda=R^{*} \lambda$ so that $R^{*} \lambda$ (and similarly $S^{*} \lambda$ ) is also a $T$-invariant probability. But $a R^{*} \lambda+\beta S^{*} \lambda=T^{*} \lambda=\lambda$. So by extremality of $\lambda$ we have $R^{*} \lambda=S^{*} \lambda=\lambda$. Thus the extremes of $K(T)$ are all in $K(R) \cap$ $K(S)$. The Krein-Mil'man theorem shows $K(T) \subset K(R) \cap K(S)$ and now the Lemma finishes the proof.

We base a second observation on the Leimma (together with the Theorem). If $R$ and $S$ commute and each is uniquely ergodic so $K(R)=K(S)=\{\mu\}$ then $T=\alpha R+\beta S$ is uniquely ergodic and $K(T)=K(R)=K(S)=\{\mu\}$.

It is not possible to derive u.m.s. in the reverse direction. That is $T$ and $R$ may be u.m.s. while $S$ fails to be u.m.s. For let $X$ be the Stone-Čech compactification of $Z_{+}$. Let $S$ be the extension to $X$ of the shift on $Z_{+}$. Let $\mu$ be a shift invariant probability on $X$ and define $R$ to be the projection $R f=(f, \mu)$. Then $T=1 / 2(R+S)$ is not only u.m.s. for we even have $T^{n}$ convergent in the strong operator topology (to $R$ ). Now $R$ itself is u.m.s. but $S$ has many invariant probabilities while only constants for invariant functions so cannot be u.m.s.

Finally we may ask what can be said if $\alpha$ and $\beta$ are functions on $X$ instead of constants. Under most circumstances $\alpha R$ and $\beta S$ no longer commute and the instances in which they do are too specialized to be of interest. We will look at one example where the coefficient functions are allowed to vary over $X$. The convex combination will fail to be u.m.s. because of stagnation caused by the coefficients. Let $X$ be the interval $[0,1]$ and define

$$
T f(x)=x f(x)+(1-x) f(x / 2) .
$$

Note that $S f=f(x / 2)$ while $R$ is the identity transformation. Thus $K(R)$ is the set of all probabilities on $X$ while $K(S)=\{\delta(0)\}$. It is easy to show that $\delta(1)$ is $T$-invariant as well as $\delta(0)$. If $T$ were u.m.s. there would be a continuous $T$-invariant function $f$ with $f(0)=0$ and $f(1)=1$. But then there would be a point $x_{0}$ in $X$ with $f\left(x_{0} / 2\right)<f\left(x_{0}\right)$.

$$
\begin{aligned}
f\left(x_{0}\right) & =T f\left(x_{0}\right)=x_{0} f\left(x_{0}\right)+\left(1-x_{0}\right) f\left(x_{0} / 2\right) \\
& <x_{0} f\left(x_{0}\right)+\left(1-x_{0}\right) f\left(x_{0}\right)=f\left(x_{0}\right) .
\end{aligned}
$$

This contradiction shows $T$ is not u.m.s.

Added in proof. The key lemma used here is an inadvertent rediscovery of a result of M. Falkowitz [7]. The example shows that Falkowitz's result is 
sharp in that the result may fail if the convex coefficients are continuous functions rather than constants.

\section{BIBLIOGR APHY}

1. B. Jamison, Ergodic decompositions induced by certain Markov operators, Trans. Amer. Math. Soc. 117 (1965), 451-468. MR $34 \# 6857$.

2. S. P. Llyod, On certain projections in spaces of continuous functions, Pacific J. Math. 13 (1963), 171-175. MR $27 \# 2845$.

3. J. C. Oxtoby, Ergodic sets, Bull. Amer. Math. Soc. 58 (1952), 116-136. MR 13, 850 .

4. M. Rosenblatt, Equicontinuous Markov operators, Teor. Verojatnost. i Primenen. 9 (1964), 205-222. MR $30 \sharp 1549$.

5. R. Sine, Geometric theory of a single Markov operator, Pacific J. Math. 27 (1968), 155-166. MR 39 \#1630.

6. - A mean ergodic theorem, Proc. Amer. Math. Soc. 24 (1970), 438-439. MR $40 \# 5825$.

7. M. Falkowitz, On finite invariant measures for Markov operators, Proc. Amer. Math. Soc. 38(1973), 553-557. MR $47 \# 880$.

DEPARTMENT OF MATHEMATICS, STATE UNIVERSITY OF NEW YORK AT ALBANY, ALBANY, NEW YORK 12222

Current address: Department of Mathematics, University of Rhode Island, Kingston, Rhode Island 02881 